\title{
Special Issue on Concussion Biomechanics in Football
}

\author{
(published online 3 November 2020)
}

This special issue is focused on the biomechanics of concussion in football. These papers are timely given increased public awareness concerning concussions in recent years, which has led to a push for more research in the area. Repetitive head impact exposure leading to long-term neurocognitive deficits is particularly concerning, with evidence for poorer outcomes associated with longer exposure. ${ }^{10}$ The study of concussion in football has broad applications both within the sport as well as more general areas of impact protection. Player safety can be improved through rule and practice structure changes informed by analysis of athlete head impact exposure. Improvements in helmets and other safety equipment can be guided by laboratory and on-field evaluations. Fundamental injury mechanisms and brain injury tolerance can be translated to enhanced safety for other sports and recreational activities, automotive environments, and military personnel. This special issue will highlight the most recent advances in football concussion biomechanics research including analysis of real-world impacts, new head impact sensor technologies, and laboratory evaluation of helmets.

The biomechanics of on-field head impacts have been analyzed through reconstructions of videorecorded impacts and wearable head impact sensors. Video recordings of National Football League (NFL) games were used to reconstruct concussive or severe impacts at the professional level. ${ }^{11}$ The videos were analyzed for pre-impact velocity and location, and impacts were recreated in the lab with anthropomorphic test devices (ATDs). The results of the reconstructions provided average linear and rotational head accelerations for concussed players as well as risk functions based on head kinematics and head injury metrics. More recently, the NFL reconstructions were reanalyzed due to inconsistencies in the original instrumentation, resulting in error in the head kinematics of concussed players. ${ }^{16}$

Wearable head impact sensors have been used to measure head impact kinematics in football since the early 1960s. ${ }^{12}$ Helmet-mounted sensors have been used to collect a large dataset of head impacts to determine head impact exposure and concussion biomechanics in collegiate and high school football players. ${ }^{3,4,18}$ Analysis of head impact exposure during different drills and event types can inform rule and practice structure changes to improve player safety. ${ }^{1,17}$ These data have also been used to evaluate concussion tolerance and risk in football players, with recent work showing that tolerance varies on an individual basis and may have a number of underlying factors. ${ }^{14}$

There are limitations and advantages to both laboratory reconstructions and wearable sensors for studying real-world head impact biomechanics. Laboratory grade instrumentation for reconstructions is highly accurate, but the impact conditions are not identical to what occurs on the field. Videos used for reconstructions are adequate for determining pre-impact velocity and in some cases helmet kinematics, but head kinematics are substantially less than what the helmet experiences during impact. ${ }^{2,8}$ Wearable sensors such as the helmet-mounted ones previously mentioned are able to capture head kinematics under realworld conditions, but the sensors vary in accuracy largely due to difficulty tightly coupling sensors to the skull. ${ }^{20}$ Relative motion between the sensor and skull often results in overprediction of head kinematics and injury risk. One solution for improved coupling and more accurate measurement of athlete head kinematics is sensors embedded in mouthguards or retainers. ${ }^{13}$

Head impact data collected on the field have been used to develop helmet evaluation methods to inform consumers about the relative performance of different models and drive improvements in helmet design. 5,15,19 These evaluations use laboratory impacts representative of on-field head impacts along with injury metrics to rank relative performance of football helmets. The injury criteria are based on head kinematics, however some more recent work has developed metrics that predict brain strain based on input head kinematics. ${ }^{6,7}$ The strain-based metrics consider the injury mechanism for concussion rather than just a kinematic variable that correlates with injury.

A comprehensive understanding of concussion biomechanics requires reliable and objective concussion diagnostic tools to differentiate injurious and noninjurious impacts. Blood biomarkers have shown 
promise for concussion diagnosis, and can be paired with biomechanical data from players for a more complete understanding of injury mechanisms. ${ }^{9} \mathrm{We}$ hope that the papers in this special issue serve as a reference and foundation for future advancements in football concussion biomechanics research.

\section{REFERENCES}

${ }^{1}$ Asken, B. M., Z. S. Brooke, T. C. Stevens, P. G. Silvestri, M. J. Graham, M. S. Jaffee, and J. R. Clugston. Drillspecific head impacts in collegiate football practice: implications for reducing "friendly fire" exposure. Ann. Biomed. Eng. 47:2094-2108, 2019.

${ }^{2}$ Bailey, A., J. Funk, D. Lessley, C. Sherwood, J. Crandall, W. Neale and N. Rose. Validation of a videogrammetry technique for analysing American football helmet kinematics. Sports Biomech. 1-23, 2018.

${ }^{3}$ Broglio, S. P., T. Surma, and J. A. Ashton-Miller. High school and collegiate football athlete concussions: a biomechanical review. Ann. Biomed. Eng. 40:37-46, 2012.

${ }^{4}$ Campolettano, E. T., S. Rowson, S. M. Duma, B. Stemper, A. Shah, J. Harezlak, L. D. Riggen, J. Mihalik, A. Brooks, and K. Cameron. Factors affecting head impact exposure in college football practices: a multi-institutional study. Ann. Biomed. Eng. 47:2086-2093, 2019.

${ }^{5}$ Funk J., J. Crandall, M. Wonnacott, C. Withnall and D. Drive: NFL linear impactor helmet test protocol. Biocore, Charlottesville, VA, accessed Feb 1: 2019, 2017.

${ }^{6}$ Gabler, L. F., J. R. Crandall, and M. B. Panzer. Development of a metric for predicting brain strain responses using head kinematics. Annals of biomedical engineering 46:972-985, 2018.

${ }^{7}$ Gabler, L. F., J. R. Crandall, and M. B. Panzer. Development of a second-order system for rapid estimation of maximum brain strain. Ann. Biomed. Eng. 47:1971-1981, 2019.

${ }^{8}$ Joodaki, H., A. Bailey, D. Lessley, J. Funk, C. Sherwood, and J. Crandall. Relative motion between the helmet and the head in football impact test. J. Biomech. Eng. 141:081006, 2019.

${ }^{9}$ McCrea, M., S. P. Broglio, T. W. McAllister, J. Gill, C. C. Giza, D. L. Huber, J. Harezlak, K. L. Cameron, M. N. Houston, and G. McGinty. Association of blood biomarkers with acute sport-related concussion in collegiate athletes: findings from the NCAA and Department of Defense CARE Consortium. JAMA Netw. Open 3:e1919771-e1919771, 2020.

${ }^{10}$ Montenigro, P. H., M. L. Alosco, B. M. Martin, D. H. Daneshvar, J. Mez, C. E. Chaisson, C. J. Nowinski, R. Au, A. C. McKee, and R. C. Cantu. Cumulative head impact exposure predicts later-life depression, apathy, executive dysfunction, and cognitive impairment in former high school and college football players. J. Neurotrauma 34:328340, 2017.

${ }^{11}$ Pellman, E. J., D. C. Viano, A. M. Tucker, I. R. Casson, and J. F. Waeckerle. Concussion in professional football: reconstruction of game impacts and injuries. Neurosurgery 53:799-812, 2003; (discussion 812-794).

${ }^{12}$ Reid, S. E., J. A. Tarkington, H. M. Epstein, and T. J. O'Dea. Brain tolerance to impact in football. Surg. Gynecol. Obstet. 133:929-936, 1971.

${ }^{13}$ Rich, A. M., T. M. Filben, L. E. Miller, B. T. Tomblin, A. R. Van Gorkom, M. A. Hurst, R. T. Barnard, D. S. Kohn, J. E. Urban, and J. D. Stitzel. Development, validation and pilot field deployment of a custom mouthpiece for head impact measurement. Ann. Biomed. Eng. 47:2109-2121, 2019.

${ }^{14}$ Rowson, S., E. T. Campolettano, S. M. Duma, B. Stemper, A. Shah, J. Harezlak, L. Riggen, J. P. Mihalik, K. M. Guskiewicz, and C. Giza. Accounting for variance in concussion tolerance between individuals: comparing head accelerations between concussed and physically matched control subjects. Ann. Biomed. Eng. 47:2048-2056, 2019.

${ }^{15}$ Rowson, S., and S. M. Duma. Development of the STAR evaluation system for football helmets: integrating player head impact exposure and risk of concussion. Ann. Biomed. Eng. 39:2130-2140, 2011.

${ }^{16}$ Sanchez, E. J., L. F. Gabler, A. B. Good, J. R. Funk, J. R. Crandall, and M. B. Panzer. A reanalysis of football impact reconstructions for head kinematics and finite element modeling. Clin. Biomech. 64:82-89, 2019.

${ }^{17}$ Stemper, B. D., A. S. Shah, J. Harezlak, S. Rowson, S. Duma, J. P. Mihalik, L. D. Riggen, A. Brooks, K. L. Cameron, and C. C. Giza. Repetitive head impact exposure in college football following an NCAA rule change to eliminate two-a-day preseason practices: a study from the NCAA-DoD CARE Consortium. Ann. Biomed. Eng. 47:2073-2085, 2019

${ }^{18}$ Stemper, B. D., A. S. Shah, J. Harezlak, S. Rowson, J. P. Mihalik, S. M. Duma, L. D. Riggen, A. Brooks, K. L. Cameron, and D. Campbell. Comparison of head impact exposure between concussed football athletes and matched controls: evidence for a possible second mechanism of sport-related concussion. Ann. Biomed. Eng. 47:2057-2072, 2019.

${ }^{19}$ Viano, D. C., C. Withnall, and D. Halstead. Impact performance of modern football helmets. Ann. Biomed. Eng. 40:160-174, 2012.

${ }^{20}$ Wu, L. C., V. Nangia, K. Bui, B. Hammoor, M. Kurt, F. Hernandez, C. Kuo, and D. B. Camarillo. In vivo evaluation of wearable head impact sensors. Ann. Biomed. Eng. 44:1234-1245, 2016.

\section{BETHANY RowSON \\ Virginia Tech \\ Electronic mail: browson@vt.edu}

\section{STEFAn M. Duma \\ Virginia Tech \\ Electronic mail: duma@vt.edu}

Publisher's Note Springer Nature remains neutral with regard to jurisdictional claims in published maps and institutional affiliations. 